\title{
THE MAXIMUM PRINCIPLE IN OPTIMAL CONTROL PROBLEMS WITH CONCENTRATED AND DISTRIBUTED DELAYS IN CONTROLS
}

\author{
G. KHARATISHVILI AND T. TADUMADZE
}

\begin{abstract}
In the present work there has been posed and studied a general nonlinear optimal problem and a quasi-linear optimal problem with fixed time and free right end. It contains absolutely continuous monotone delays in phase coordinates and absolutely continuous monotone and distributed delays in controls. For these problems the necessary and, respectively, sufficient conditions of optimality in the form of the maximum principle have been proved.
\end{abstract}

1. Statement of the problem. The maximum principle. Let $O_{1} \subset$ $R^{n}, O_{2} \subset R^{r}$ be open sets and $S=\left[-s_{1}, 0\right] \times \cdots \times\left[-s_{k}, 0\right], s_{k}>\cdots>$ $s_{1}>0$. Let an $n$-dimensional vector-function $f\left(t, x_{1}, \ldots, x_{s}, u_{1}, \ldots, u_{v+k}\right)$ with fixed $t \in I=\left[0, T_{0}\right]$ be continuously differentiable with respect to $\left(x_{1}, \ldots, x_{s}, u_{1}, \ldots, u_{\nu+k}\right) \in O_{1}^{s} \times O_{2}^{\nu+k}$. For fixed $\left(x_{1}, \ldots, x_{s}, u_{1}, \ldots, u_{\nu+k}\right) \in$ $O_{1}^{s} \times O_{2}^{\nu+k}$ let this function be measurable with respect to $t \in I$, like the matrix-functions $f_{x_{i}}, i=1, \ldots, s, f_{u_{j}}, j=1, \ldots, \nu+k$. For each pair of compacts $K \subset O_{1}, M \subset O_{2}$ let there exist a function $m(t)=m_{K, M}(t)$ summable on $I$ such that

$$
\begin{gathered}
\left|f\left(t, x_{1}, \ldots, x_{s}, u_{1}, \ldots, u_{\nu+k}\right)\right|+\sum_{i=1}^{s}\left|f_{x_{i}}\right|+\sum_{i=1}^{\nu+k}\left|f_{u_{i}}\right| \leq m(t), \\
\forall\left(t, x_{1}, \ldots, x_{s}, u_{1}, \ldots, u_{\nu+k}\right) \in I \times K^{s} \times M^{\nu+k} .
\end{gathered}
$$

Let now the functions $\tau_{i}(t), i=1, \ldots, s, \theta_{j}(t), j=1, \ldots, \nu, t \in I$, be absolutely continuous, satisfy the conditions $\tau_{i}(t) \leq t, \dot{\tau}_{i}(t)>0, i=$ $1, \ldots, s, \theta_{j}(t) \leq t, \dot{\theta}_{j}(t)>0, j=1, \ldots, \nu$, and $\tau=\min \left(\tau_{1}(0), \ldots, \tau_{s}(0)\right)$, $\theta=\min \left(\theta_{1}(0), \ldots, \theta_{\nu}(0),-s_{k}\right) ; U_{1} \subset O_{2}, N \subset O_{1}$ be convex sets and $\Omega_{1}=\Omega\left(U_{1}\right)=\left\{u \in L_{\infty} \mid u(t) \in U_{1}, t \in\left[\theta, T_{0}\right]\right.$, the closure of $u\left(\left[\theta, T_{0}\right]\right)$ is

1991 Mathematics Subject Classification. 49K25.

Key words and phrases. Maximum principle, optimal control problem, delays in controls, sufficient conditions for optimality. 
compact and belongs to $O_{2}$ \}. Let $L_{\infty}$ be a space of essentially bounded measurable functions $u:\left[\theta, T_{0}\right] \rightarrow R^{r}$ with the norm $\|u\|=\operatorname{ess} \sup |u(t)|$, $t \in\left[\theta, T_{0}\right] ; G=\left\{\varphi \in E_{\varphi} \mid \varphi(t) \in N, t \in[\tau, 0]\right\}, E_{\varphi}$ be the space of piecewise continuous functions $\varphi:[\tau, 0] \rightarrow R^{n}$ with a finite number of discontinuity points of the first kind with the norm $\|\varphi\|=\sup |\varphi(t)|, t \in[\tau, 0]$, and let $q^{i}\left(t, x_{0}, x_{1}\right),\left(t, x_{0}, x_{1}\right) \in I \times O_{1}^{2}, i=0, \ldots, l$, be continuously differentiable scalar functions.

Definition 1. We shall call the element $\zeta=\left(T, x_{0}, \varphi, u\right) \in I \times O_{1} \times G \times \Omega_{1}$ admissible if the corresponding solution $x(t)=x(t ; \zeta)$ of the system

$$
\begin{gathered}
\dot{x}(t)=\int_{S} f\left(t, x\left(\tau_{1}(t)\right), \ldots, x\left(\tau_{s}(t)\right), u\left(\theta_{1}(t)\right), \ldots, u\left(\theta_{\nu}(t)\right), u\left(t+s_{1}\right), \ldots\right. \\
\left.\ldots, u\left(t+s_{k}\right)\right) d S, \quad t \in[0, T] \subset I, u \in \Omega_{1}
\end{gathered}
$$

(here and in what follows $d S$ stands for $d s_{1} \ldots d s_{k}$ ), with the initial condition

$$
x(t)=\varphi(t), \quad t \in[\tau, 0), x(0)=x_{0},
$$

is defined on the interval $[0, T]$ and satisfies the condition

$$
q^{i}\left(T, x_{0}, x(T)\right)=0, \quad i=1, \ldots, l .
$$

We shall denote a set of admissible elements by $\Delta_{1}$.

Definition 2. We call the element $\widetilde{\zeta}=\left(\widetilde{T}, \widetilde{x}_{0}, \widetilde{\varphi}, \widetilde{u}\right) \in \Delta_{1}, \widetilde{T} \in\left(0, T_{0}\right)$ optimal if there exists a number $\delta>0$ such that for each element $\zeta=$ $\left(T, x_{0}, \varphi, u\right) \in \Delta_{1}$ satisfying the condition

$$
|T-\widetilde{T}|+\left|x_{0}-\widetilde{x}_{0}\right|+\|\varphi-\widetilde{\varphi}\|+\|u-\widetilde{u}\| \leq \delta,
$$

the inequality

$$
q^{0}\left(\widetilde{T}, \widetilde{x}_{0}, \widetilde{x}(\widetilde{T})\right) \leq q^{0}\left(T, x_{0}, x(T)\right)
$$

is fulfilled, where $\widetilde{x}(t)=x(t ; \widetilde{\zeta}), x(t)=x(t ; \zeta)$.

The optimal problem consists in finding the optimal elements.

Theorem 1 (The necessary conditions of optimality). Let $\widetilde{\zeta} \in \Delta_{1}$ be an optimal element and $t=\widetilde{T}$ be a Lebesgue point of the function

$$
\begin{gathered}
\widetilde{f}(t)=\int_{S} f\left(t, \widetilde{x}\left(\tau_{1}(t)\right), \ldots, \widetilde{x}\left(\tau_{s}(t)\right), \widetilde{u}\left(\theta_{1}(t)\right), \ldots\right. \\
\left.\ldots, \widetilde{u}\left(\theta_{\nu}(t)\right), \widetilde{u}\left(t+s_{1}\right), \ldots, \widetilde{u}\left(t+s_{k}\right)\right) d S
\end{gathered}
$$


Then there exist a nonzero vector $\pi=\left(\pi_{0}, \ldots, \pi_{l}\right), \pi_{0} \leq 0$, and a solution $\psi(t), t \in[0, \widetilde{T}]$ of the system

$$
\dot{\psi}(t)=-\sum_{j=1}^{s} \psi\left(\gamma_{j}(t)\right) \widetilde{f}_{x_{j}}\left(\gamma_{j}(t)\right) \dot{\gamma}_{j}(t), \quad t \in[0, \widetilde{T}], \psi(t)=0, \quad t>\widetilde{T},
$$

such that the following conditions are fulfilled:

1. the integral maximum principle:

$$
\begin{gathered}
\sum_{j=1}^{s} \int_{\tau_{j}(0)}^{0} \psi\left(\gamma_{j}(t)\right) \widetilde{f}_{x_{j}}\left(\gamma_{j}(t)\right) \dot{\gamma}_{j}(t) \widetilde{\varphi}(t) d t \geq \\
\geq \sum_{j=1}^{s} \int_{\tau_{j}(0)}^{0} \psi\left(\gamma_{j}(t)\right) \widetilde{f}_{x_{j}}\left(\gamma_{j}(t)\right) \dot{\gamma}_{j}(t) \varphi(t) d t, \quad \forall \varphi \in G, \\
\int_{0}^{\widetilde{T}} \psi(t)\left\{\sum_{i=1}^{\nu} \widetilde{f}_{u_{i}}(t) \widetilde{u}\left(\theta_{i}(t)\right)+\sum_{i=1}^{k} \int_{S} \widetilde{f}_{u_{\nu+i}}\left(t, s_{1}, \ldots, s_{k}\right) \times\right. \\
\left.\times \widetilde{u}\left(t+s_{i}\right) d S\right\} d t \geq \int_{0}^{\widetilde{T}} \psi(t)\left\{\sum_{i=1}^{\nu} \widetilde{f}_{u_{i}}(t) u\left(\theta_{i}(t)\right)+\right. \\
\left.+\sum_{i=1}^{k} \int_{S} \widetilde{f}_{u_{\nu+i}}\left(t, s_{1}, \ldots, s_{k}\right) u\left(t+s_{i}\right) d S\right\} d t, \quad \forall u \in \Omega_{1} ;
\end{gathered}
$$

2. the conditions of transversality:

$$
\pi \widetilde{Q}_{t}=-\psi(\widetilde{T}) \widetilde{f}(\widetilde{T}), \quad \pi \widetilde{Q}_{x_{0}}=-\psi(0), \quad \pi \widetilde{Q}_{x_{1}}=\psi(\widetilde{T}) .
$$

Here $Q=\left(q^{0}, \ldots, q^{l}\right)$, the tilde sign over $Q$ means that the corresponding gradient is calculated at the point $\left(\widetilde{T}, \widetilde{x}_{0}, \widetilde{x}(\widetilde{T})\right), \gamma_{j}(t)$ is the inverse of the function $\tau_{j}(t)$,

$$
\begin{gathered}
\widetilde{f}_{x_{j}}(t)=\int_{S} \widetilde{f}_{x_{j}}\left(t, s_{1}, \ldots, s_{k}\right) d S \\
\widetilde{f}_{x_{j}}\left(t, s_{1}, \ldots, s_{k}\right)=f_{x_{j}}\left(t, \widetilde{x}\left(\tau_{1}(t)\right), \ldots, \widetilde{x}\left(\tau_{s}(t)\right), \widetilde{u}\left(\theta_{1}(t)\right), \ldots\right. \\
\left.\ldots, \widetilde{u}\left(\theta_{\nu}(t)\right), \widetilde{u}\left(t+s_{1}\right), \ldots, \widetilde{u}\left(t+s_{k}\right)\right) .
\end{gathered}
$$

Remark 1. If the rank of the matrix $\left(\widetilde{Q}_{t}, \widetilde{Q}_{x_{0}}, \widetilde{Q}_{x_{1}}\right)$ is equal to $1+l$, then $\psi(t) \not \equiv 0$.

Consider now the problem with a fixed time and a free right end:

$$
\dot{x}(t)=\sum_{i=1}^{s} A_{i}(t) x\left(\tau_{i}(t)\right)+\int_{S} f\left(t, u\left(\theta_{1}(t)\right), \ldots\right.
$$




$$
\begin{gathered}
\left.\ldots, u\left(\theta_{\nu}(t)\right), u\left(t+s_{1}\right), \ldots, u\left(t+s_{k}\right)\right) d S \\
x(t)=\varphi(t), \quad t \in[\tau, 0), \quad x(0)=x_{0}, \quad \varphi \in G, \\
I(\zeta)=\int_{0}^{T_{0}}\left[g\left(t, x\left(\tau_{1}(t)\right), \ldots, x\left(\tau_{s}(t)\right)\right)+\int_{S} f^{0}\left(t, u\left(\theta_{1}(t)\right), \ldots\right.\right. \\
\left.\left.\ldots, u\left(\theta_{\nu}(t)\right), u\left(t+s_{1}\right), \ldots, u\left(t+s_{k}\right)\right) d S\right] d t
\end{gathered}
$$

where $A_{i}(t), i=1, \ldots, s, t \in I$, are the summable matrix-functions; the function $f\left(t, u_{1}, \ldots, u_{\nu+k}\right)$ with fixed $t \in I$ is continuous with respect to $\left(u_{1}, \ldots, u_{\nu+k}\right) \in O_{2}^{\nu+k}$, and with fixed $\left(u_{1}, \ldots, u_{\nu+k}\right) \in O_{2}^{\nu+k}$ it is measurable with respect to $t \in I$; for each compact $M \subset O_{2}$ there exists a summable function $m_{1}(t)=m_{M}(t)$ such that

$$
\left|f\left(t, u_{1}, \ldots, u_{\nu+k}\right)\right| \leq m_{1}(t), \quad \forall\left(t, u_{1}, \ldots, u_{\nu+k}\right) \in I \times M^{\nu+k} ;
$$

the function $g\left(t, x_{1}, \ldots, x_{s}\right)$ with fixed $t \in I$ is continuously differentiable and convex with respect to $\left(x_{1}, \ldots, x_{s}\right) \in O_{3}^{n s}$, with fixed $\left(x_{1}, \ldots, x_{s}\right) \in O_{3}^{n s}$ it is measurable with respect to $t \in I$, and for each compact $K \subset O_{3}$ there exists a summable function $m_{2}(t)=m_{k}(t)$ such that

$$
\left|g\left(t, x_{1}, \ldots, x_{s}\right)\right| \leq m_{2}(t), \forall\left(t, x_{1}, \ldots, x_{s}\right) \in I \times O_{3}^{n s} ;
$$

$O_{3} \subset R^{n}$ is a convex open set; the scalar function $f^{0}$ satisfies some conditions like $f$; moreover, $\Omega_{2}=\Omega\left(U_{2}\right)$, where $U_{3} \subset O_{2}$ is an arbitrary set and $x_{0} \in R^{n}$ is a given point.

Definition 3. An element $\widetilde{\zeta}=(\widetilde{\varphi}, \widetilde{u}) \in \Delta_{2}=G \times \Omega_{2}$ is called optimal if for each $\zeta \in \Delta_{2}$ the inequality

$$
I(\widetilde{\zeta}) \leq I(\zeta)
$$

is fulfilled.

Theorem 2. For an element $\widetilde{\zeta} \in \Delta_{2}$ to be optimal, it suffices to fulfill the following conditions:

$$
\begin{gathered}
\sum_{j=1}^{s} \int_{\tau_{j}(0)}^{0}\left[\psi\left(\gamma_{j}(t)\right) A_{j}\left(\gamma_{j}(t)\right)-\widetilde{g}_{x_{j}}\left(\gamma_{j}(t)\right)\right] \dot{\gamma}_{j}(t) \widetilde{\varphi}(t) d t \geq \\
\geq \sum_{j=1}^{s} \int_{\tau_{j}(0)}^{0}\left[\psi\left(\gamma_{j}(t)\right) A_{j}\left(\gamma_{j}(t)\right)-\widetilde{g}_{x_{j}}\left(\gamma_{j}(t)\right)\right] \dot{\gamma}_{j}(t) \varphi(t) d t, \quad \forall \varphi \in G, \\
\int_{0}^{T_{0}}\left[\psi(t) f(t, \widetilde{u}(\cdot))-f^{0}(t, \widetilde{u}(\cdot))\right] d t \geq \int_{0}^{T_{0}}[\psi(t) f(t, u(\cdot))- \\
\left.-f^{0}(t, u(\cdot))\right] d t, \quad \forall u \in \Omega_{2}
\end{gathered}
$$


Here

$$
f(t, u(\cdot))=\int_{S} f\left(t, u\left(\theta_{1}(t)\right), \ldots, u\left(\theta_{\nu}(t)\right), u\left(t+s_{1}\right), \ldots, u\left(t+s_{k}\right)\right) d S,
$$

$f^{0}(t, u(\cdot))$ is defined analogously; $\widetilde{g}_{x_{j}}(t)=g_{x_{j}}\left(t, \widetilde{x}\left(\tau_{1}(t)\right), \ldots, \widetilde{x}\left(\tau_{s}(t)\right)\right)$ and $\psi(t)$ is the solution of the system

$$
\begin{gathered}
\dot{\psi}(t)=\sum_{j=1}^{s}\left[\psi_{0}\left(\gamma_{j}(t)\right) \widetilde{g}_{x_{j}}\left(\gamma_{j}(t)\right)-\psi\left(\gamma_{j}(t)\right) A_{j}\left(\gamma_{j}(t)\right)\right] \dot{\gamma}_{j}(t), \quad t \in\left[0, T_{0}\right] \\
\psi(t)=0, \quad t \geq T_{0}, \quad \psi_{0}(t)=1, \quad t \in\left[0, T_{0}\right], \quad \psi_{0}(t)=0, \quad t>T_{0} .
\end{gathered}
$$

Remark 2. Theorems 1 and 2 are valid in the case where $\theta_{j}(t), t \in I, j=$ $1, \ldots, \nu$, are piecewise absolutely continuous functions (with a finite number of discontinuity points) satisfying the conditions $\theta_{j}(t) \leq t$ and $\dot{\theta}_{j}(t)>0$.

\section{Proof of Theorem 1.}

2.1. Continuous dependence and differentiability of the solution. Let $\widetilde{\zeta}=\left(\widetilde{T}, \widetilde{x}_{0}, \widetilde{\varphi}, \widetilde{u}\right) \in \Delta_{1}, \widetilde{T}<T_{0}$ be an optimal element of the problem $(2)-(5)$ and let $\widetilde{x}(t)=x(t ; \widetilde{\zeta}), t \in[0, \widetilde{T}]$ be the corresponding solution of system (2).

Lemma 1. For each $\varepsilon>0$ there exists a number $\delta=\delta(\varepsilon)>0$ such that for each $\mu=\left(x_{0}, \varphi, u\right) \in O_{1} \times G \times \Omega_{1}$ satisfying the condition

$$
\left|x_{0}-\widetilde{x}_{0}\right|+\|\varphi-\widetilde{\varphi}\|+\|u-\widetilde{u}\| \leq \delta
$$

the system

$$
\begin{gathered}
\dot{x}(t)=\int_{S} f\left(t, x\left(\tau_{1}(t)\right), \ldots, x\left(\tau_{s}(t)\right), u\left(\theta_{1}(t)\right), \ldots\right. \\
\left.\ldots, u\left(\theta_{\nu}(t)\right), u\left(t+s_{1}\right), \ldots, u\left(t+s_{k}\right)\right) d S \\
x(t)=\varphi(t), \quad t \in[\tau, 0), \quad x(0)=x_{0}
\end{gathered}
$$

has the solution $x(t ; \mu)$ which is defined on $[0, \widetilde{T}+\delta] \subset I$. In this case if $\mu_{i}=\left(x_{0}^{i}, \varphi_{i}, u_{i}\right), i=1,2$, satisfy the condition (15), then

$$
\left|x\left(t ; \mu_{1}\right)-x\left(t ; \mu_{2}\right)\right| \leq \varepsilon, \quad t \in[0, \widetilde{T}+\delta] .
$$

Proof. We rewrite the system (16) in the form

$$
\begin{gathered}
\dot{x}(t)=\tilde{f}\left(t, x\left(\tau_{1}(t)\right), \ldots, x\left(\tau_{s}(t)\right)\right)+\delta f\left(t, x\left(\tau_{1}(t)\right), \ldots, x\left(\tau_{s}(t)\right)\right), \\
x(t)=\varphi(t), \quad t \in[\tau, 0), x(0)=x_{0},
\end{gathered}
$$

where

$$
\widetilde{f}\left(t, x_{1}, \ldots, x_{s}\right)=\int_{S} f\left(t, x_{1}, \ldots, x_{s}, \widetilde{u}\left(\theta_{1}(t)\right), \ldots\right.
$$




$$
\begin{gathered}
\left.\ldots, \widetilde{u}\left(\theta_{\nu}(t)\right), \widetilde{u}\left(t+s_{1}\right), \ldots, \widetilde{u}\left(t+s_{k}\right)\right) d S \\
\delta f\left(t, x_{1}, \ldots, x_{s}\right)=\int_{S}\left[f \left(t, x_{1}, \ldots, x_{s}, u\left(\theta_{1}(t)\right), \ldots\right.\right. \\
\left.\ldots, u\left(\theta_{\nu}(t)\right), u\left(t+s_{1}\right), \ldots, u\left(t+s_{k}\right)\right)-f\left(t, x_{1}, \ldots, x_{s}, \widetilde{u}\left(\theta_{1}(t)\right), \ldots\right. \\
\left.\left.\ldots, \widetilde{u}\left(\theta_{\nu}(t)\right), \widetilde{u}\left(t+s_{1}\right), \ldots, \widetilde{u}\left(t+s_{k}\right)\right)\right] d S .
\end{gathered}
$$

Let $M_{0} \subset O_{2}$ be the closure of the set $\widetilde{u}\left(\left[\theta, T_{0}\right]\right)$. Then there exists a number $\delta>0$ such that the functions $u \in V_{\delta}=\left\{u \in \Omega_{1} \mid\|u-\widetilde{u}\| \leq \delta\right\}$ for almost all $t \in\left[\theta, T_{0}\right]$ take values from the compact $M_{1} \subset O_{2}$ containing some neighborhood of the compact $M_{0}$.

Let $K_{0} \subset O_{1}$ be a compact containing some neighborhood of the set $\{\widetilde{x}(t) \mid t \in[\tau, \widetilde{T}]\}$.

We shall estimate $\delta f\left(t, x_{1}, \ldots, x_{s}\right)$ for each $\left(x_{1}, \ldots, x_{s}\right) \in K_{0}^{s}, u \in V_{\delta}$. It is not difficult to notice that

$$
\begin{gathered}
|\delta f| \leq \int_{S}\left\{\int_{0}^{1} \mid \frac{d}{d \xi} f\left(t, x_{1}, \ldots, x_{s}, \widetilde{u}\left(\theta_{1}(t)\right)+\xi \delta u\left(\theta_{1}(t)\right), \ldots\right.\right. \\
\ldots, \widetilde{u}\left(\theta_{\nu}(t)\right)+\xi \delta u\left(\theta_{\nu}(t)\right), \widetilde{u}\left(t+s_{1}\right)+\xi \delta u\left(t+s_{1}\right), \ldots \\
\left.\left.\ldots, \widetilde{u}\left(t+s_{k}\right)+\xi \delta u\left(t+s_{k}\right)\right) \mid d \xi\right\} d S
\end{gathered}
$$

where $\delta u(t)=u(t)-\widetilde{u}(t)$.

It is clear that $\widetilde{u}+\xi \delta u \in V_{\delta}$ and hence, $\widetilde{u}(t)+\xi \delta u(t) \in M_{1}$ for almost all $t \in\left[\theta, T_{0}\right]$.

Taking (1) into consideration, we obtain

$$
\begin{gathered}
|\delta f| \leq \int_{S}\left\{\int _ { 0 } ^ { 1 } \left[\sum_{j=1}^{\nu} \mid f_{u_{i}}\left(t, x_{1}, \ldots, x_{s}, \widetilde{u}\left(\theta_{1}(t)\right)+\xi \delta u\left(\theta_{1}(t)\right), \ldots\right.\right.\right. \\
\ldots, \widetilde{u}\left(\theta_{\nu}(t)\right)+\xi \delta u\left(\theta_{\nu}(t)\right), \widetilde{u}\left(t+s_{1}\right)+\xi \delta u\left(t+\xi_{1}\right), \ldots \\
\left.\ldots, \widetilde{u}\left(t+s_{k}\right)+\xi \delta u\left(t+\xi_{k}\right)\right)|| \delta u\left(\theta_{j}(t)\right) \mid+ \\
+\sum_{j=1}^{k} \mid f_{u_{\nu+j}}\left(t, x_{1}, \ldots, x_{s}, \widetilde{u}\left(\theta_{1}(t)\right)+\xi \delta u\left(\theta_{1}(t)\right), \ldots, \widetilde{u}\left(\theta_{\nu}(t)\right)+\right. \\
\left.+\xi \delta u\left(\theta_{\nu}(t)\right), \widetilde{u}\left(t+s_{1}\right)+\xi \delta u\left(t+s_{1}\right), \ldots, \widetilde{u}\left(t+s_{k}\right)+\xi \delta u\left(t+s_{k}\right)\right) \mid \times \\
\left.\left.\times\left|\delta u\left(t+s_{j}\right)\right|\right] d \xi\right\} d S \leq\left(s_{1} \ldots s_{k}\right) m_{k_{0}, M_{0}}(t)\|u-\widetilde{u}\| .
\end{gathered}
$$

Thus for each $\left(x_{1}, \ldots, x_{s}\right) \in K_{0}^{s}, u \in V_{\delta}, t^{\prime}, t^{\prime \prime} \in I$ we have

$$
\left|\int_{t^{\prime}}^{t^{\prime \prime}} \delta f\left(t, x_{1}, \ldots, x_{s}\right) d t\right| \leq c_{0} \delta
$$


Now it is not difficult to see that

$$
\int_{I}\left\{\left|\delta f\left(t, x_{1}, \ldots, x_{s}\right)\right|+\sum_{j=1}^{s}\left|\delta f_{x_{j}}\right|\right\} d t \leq c_{1}=\text { const. }
$$

The inequalities (17) and (18) allow us to conclude that Lemma 1 implies Theorem 2.8 (see [1], p.52).

Now let us introduce the set $V_{1}=\left\{\delta \mu=\left(\delta x_{0}, \delta \varphi, \delta u\right) \mid \delta x_{0} \in O_{1}-\widetilde{x}_{0}\right.$, $\delta \varphi \in G-\widetilde{\varphi}, \delta u \in \Omega-\widetilde{u},\left|\delta x_{0}\right| \leq c=$ const $\left.,\|\delta \varphi\| \leq c,\|\delta u\| \leq c\right\}$.

Lemma 2. There exist numbers $\varepsilon_{0}>0, \delta_{0}>0$ such that for each $\varepsilon \in\left[0, \varepsilon_{0}\right]$ and $\delta \mu \in V_{1}$ the solution $x(t ; \varepsilon \delta \mu)=x(t ; \widetilde{\mu}+\varepsilon \delta \mu)$, $\widetilde{\mu}=\left(\widetilde{x}_{0}, \widetilde{\varphi}, \widetilde{u}\right)$, is defined on $\left[0, \widetilde{T}+\delta_{0}\right]$, and

$$
\lim _{\varepsilon \rightarrow 0} x(t ; \varepsilon \delta \mu)=x(t ; \widetilde{\mu}) \text { is uniform over }(t, \delta \mu) \in\left[0, \widetilde{T}+\delta_{0}\right] \times V_{1} \text {. }
$$

\section{Moreover}

$$
x(t ; \varepsilon \delta \mu)=\widetilde{x}(t)+\varepsilon \delta x(t ; \delta \mu)+R(t ; \varepsilon \delta \mu), \quad t \in\left[0, \widetilde{T}+\delta_{0}\right],
$$

where $\widetilde{x}(t)=x(t ; \widetilde{\mu})$,

$$
\begin{gathered}
\delta x(t ; \delta \mu)=Y(0 ; t) \delta x_{0}+\sum_{j=1}^{s} \int_{\tau_{j}(0)}^{0} Y\left(\gamma_{j}(\xi) ; t\right) \widetilde{f}_{x_{j}}\left(\gamma_{j}(\xi)\right) \times \\
\times \dot{\gamma}_{j}(\xi) \delta \varphi(\xi) d \xi+\int_{0}^{t} Y(\xi ; t)\left\{\sum_{j=1}^{\nu} \widetilde{f}_{u_{j}}(\xi) \delta u\left(\theta_{j}(\xi)\right)+\right. \\
\left.+\sum_{j=1}^{k} \int_{S} \widetilde{f}_{u_{\nu+j}}\left(\xi, s_{1}, \ldots, s_{k}\right) \delta u\left(\xi+s_{j}\right) d S\right\} d \xi \\
\lim _{\varepsilon \rightarrow 0} \varepsilon^{-1} R(t ; \varepsilon \delta \mu)=0 \text { is uniform over }(t, \delta \mu) \in\left[0, \widetilde{T}+\delta_{0}\right] \times V_{1} .
\end{gathered}
$$

Here $Y(\xi ; t), \xi, t \in I$ is a matrix function satisfying both the matrix equation

$$
\frac{\partial Y(\xi ; t)}{\partial \xi}+\sum_{j=1}^{s} Y\left(\gamma_{j}(\xi) ; t\right) \widetilde{f}_{x_{j}}\left(\gamma_{j}(\xi)\right) \dot{\gamma}_{j}(\xi)=0, \quad 0 \leq \xi \leq t
$$

and the condition

$$
Y(\xi ; t)=\left\{\begin{array}{l}
E, \quad \xi=t \\
0, \quad \xi>t
\end{array}\right.
$$


Proof. The first part of the Lemma is a simple corollary of Lemma 1.

It is easily seen that the function $\Delta x(t)=x(t)-\widetilde{x}(t)$, where $x(t)=$ $x(t ; \varepsilon \delta \mu)$, satisfies the system

$$
\begin{aligned}
& \quad \frac{d}{d t} \Delta x(t)=\int_{S} f\left(t, x\left(\tau_{1}(t), \ldots, x\left(\tau_{s}(t), \widetilde{u}\left(\theta_{1}(t)\right)+\right.\right.\right. \\
& +\varepsilon \delta u\left(\theta_{1}(t)\right), \ldots, \widetilde{u}\left(\theta_{\nu}(t)\right)+\varepsilon \delta u\left(\theta_{\nu}(t)\right), \widetilde{u}\left(t+s_{1}\right)+ \\
& \left.+\varepsilon \delta u\left(t+s_{1}\right), \ldots, \widetilde{u}\left(t+s_{k}\right)+\varepsilon \delta u\left(t+s_{k}\right)\right) d S-\widetilde{f}(t),
\end{aligned}
$$

and the initial condition

$$
\Delta x(t)=\varepsilon \delta \varphi(t), \quad t \in[\tau, 0), \quad \Delta x(0)=\varepsilon \delta x_{0} .
$$

We can rewrite the system (22) in the form

$$
\begin{aligned}
& \frac{d}{d t} \Delta x(t)=\sum_{j=1}^{s} \widetilde{f}_{x_{j}}(t) \Delta x\left(\tau_{j}(t)\right)+\varepsilon \sum_{j=1}^{\nu} \widetilde{f}_{u_{j}}(t) \delta u\left(\theta_{j}(t)\right)+ \\
& \quad+\varepsilon \sum_{j=1}^{k} \int_{S} f_{u_{\nu+j}}\left(t, s_{1}, \ldots, s_{k}\right) \delta u\left(t+s_{j}\right) d S+\Gamma(t ; \varepsilon \delta \mu),
\end{aligned}
$$

where

$$
\begin{gathered}
\Gamma(t ; \varepsilon \delta \mu)=\int_{S}\left[f \left(t, x\left(\tau_{1}(t)\right), \ldots, x\left(\tau_{s}(t)\right), \widetilde{u}\left(\theta_{1}(t)\right)+\varepsilon \delta u\left(\theta_{1}(t)\right), \ldots\right.\right. \\
\left.\ldots, \widetilde{u}\left(\theta_{\nu}(t)\right)+\varepsilon \delta u\left(\theta_{\nu}(t)\right), \widetilde{u}\left(t+s_{1}\right)+\varepsilon \delta u\left(t+s_{1}\right), \ldots, \widetilde{u}\left(t+s_{k}\right)\right)+ \\
\left.+\varepsilon \delta u\left(t+s_{k}\right)\right)-\widetilde{f}(t)-\sum_{j=1}^{s} \widetilde{f}_{x_{j}}(t) \Delta x\left(\tau_{j}(t)\right)-\varepsilon \sum_{j=1}^{\nu} f_{u_{j}}(t) \times \\
\left.\times \delta u\left(\theta_{j}(t)\right)-\varepsilon \sum_{j=1}^{k} \widetilde{f}_{u_{\nu+j}}\left(t, s_{1}, \ldots, s_{k}\right) \delta u\left(t+s_{j}\right)\right] d S .
\end{gathered}
$$

By means of the Cauchy formula the solution of the system (23) can be represented in the form

$$
\begin{gathered}
\Delta x(t)=\varepsilon\left\{Y(0 ; t) \delta x_{0}+\sum_{j=1}^{s} \int_{\tau_{j}(0)}^{0} Y\left(\gamma_{j}(\xi) ; t\right) \widetilde{f}_{x_{j}}\left(\gamma_{j}(\xi)\right) \times\right. \\
\times \dot{\gamma}_{j}(\xi) \delta \varphi(\xi) d \xi+\int_{0}^{t} Y(\xi ; t)\left[\sum_{j=1}^{\nu} \widetilde{f}_{u_{j}}(\xi) \delta u\left(\theta_{j}(\xi)\right)+\right. \\
\left.\left.\quad+\sum_{j=1}^{k} \int_{S} \widetilde{f}_{u_{\nu+j}}\left(\xi ; s_{1}, \ldots, s_{k}\right) \delta u\left(\xi+s_{j}\right) d S\right] d \xi\right\}+
\end{gathered}
$$




$$
+\int_{0}^{t} Y(\xi ; t) \Gamma(\xi) d \xi=\varepsilon \delta x(t ; \varepsilon \delta \mu)+R(t ; \varepsilon \delta \mu) .
$$

Now we shall estimate $R$. We have

$$
\begin{gathered}
|R(t ; \varepsilon \delta \mu)| \leq\|Y\| \int_{0}^{\widetilde{T}+\delta_{0}}|\Gamma(t ; \varepsilon \delta \mu)| d t \leq \\
\leq\|Y\| \int_{0}^{\widetilde{T}+\delta_{0}}\left\{\int _ { S } \left[\int_{0}^{1} \mid \frac{d}{d \xi} f\left(t, \widetilde{x}\left(\tau_{1}(t)\right)+\xi \Delta x\left(\tau_{1}(t)\right), \ldots\right.\right.\right. \\
\ldots, \widetilde{x}\left(\tau_{s}(t)\right)+\xi \Delta x\left(\tau_{s}(t)\right), \widetilde{u}\left(\theta_{1}(t)\right)+\varepsilon \xi \delta u\left(\theta_{1}(t)\right), \ldots \\
\ldots, \widetilde{u}\left(\theta_{\nu}(t)\right)+\varepsilon \xi \delta u\left(\theta_{\nu}(t)\right), \widetilde{u}\left(t+s_{1}\right)+\varepsilon \xi \delta u\left(t+s_{1}\right), \ldots \\
\left.\ldots, \widetilde{u}\left(t+s_{k}\right)+\varepsilon \xi \delta u\left(t+s_{k}\right)\right)-\sum_{j=1}^{s} \widetilde{f}_{x_{j}}(t) \Delta x\left(\tau_{j}(t)\right)- \\
\left.\left.-\varepsilon\left(\sum_{j=1}^{\nu} \widetilde{f}_{u_{j}}(t) \delta u\left(\theta_{j}(t)\right)+\sum_{j=1}^{k} \widetilde{f}_{u_{\nu+j}}\left(t, s_{1}, \ldots, s_{k}\right) \delta u\left(t+s_{j}\right)\right) \mid d \xi\right] d S\right\} d t
\end{gathered}
$$

where

$$
\Delta x(t)=x(t)-\widetilde{x}(t), \quad\|Y\|=\max _{\xi, t \in\left[0, \widetilde{T}+\delta_{0}\right]}|Y(\xi ; t)| .
$$

Differentiating the integrand with respect to $\xi$ and grouping corresponding terms we get

$$
|R(t ; \varepsilon \delta \mu)| \leq \varepsilon \sigma_{1}(\varepsilon \delta \mu)+\|\Delta x\| \sigma_{2}(\varepsilon \delta \mu)
$$

with

$$
\begin{gathered}
\|\Delta x\|=\max _{0 \leq t \leq \widetilde{T}+\delta_{0}}|\Delta x(t)|, \\
\sigma_{1}(\varepsilon \delta \mu)=c\|Y\| \int_{0}^{\widetilde{T}+\delta_{0}}\left\{\int _ { S } \left[\int _ { 0 } ^ { 1 } \left[\sum_{j=1}^{\nu} \mid f_{u_{j}}\left(t, \widetilde{x}\left(\tau_{1}(t)\right)+\xi \Delta x\left(\tau_{1}(t)\right), \ldots\right.\right.\right.\right. \\
\ldots, \widetilde{x}\left(\tau_{s}(t)\right)+\xi \Delta x\left(\tau_{s}(t)\right), \widetilde{u}\left(\theta_{1}(t)\right)+\varepsilon \xi \delta u\left(\theta_{1}(t)\right), \ldots, \widetilde{u}\left(\theta_{\nu}(t)\right)+ \\
\left.+\varepsilon \xi \delta u\left(\theta_{\nu}(t)\right), \widetilde{u}\left(t+s_{1}\right)+\varepsilon \xi \delta u\left(t+s_{1}\right), \ldots, \widetilde{u}\left(t+s_{k}\right)+\varepsilon \xi \delta u\left(t+s_{k}\right)\right)- \\
-\widetilde{f}_{u_{j}}(t)\left|+\sum_{j=1}^{k}\right| \widetilde{f}_{u_{\nu+j}}\left(t, \widetilde{x}\left(\tau_{1}(t)\right)+\xi \Delta x\left(\tau_{1}(t)\right), \ldots, \widetilde{x}\left(\tau_{s}(t)\right)+\right. \\
+\xi \Delta x\left(\tau_{s}(t)\right), \widetilde{u}\left(\theta_{1}(t)\right)+\varepsilon \xi \delta u\left(\theta_{1}(t)\right), \ldots, \widetilde{u}\left(\theta_{\nu}(t)\right)+\varepsilon \xi \delta u\left(\theta_{\nu}(t)\right), \widetilde{u}\left(t+s_{1}\right)+ \\
\left.\left.\left.\left.+\varepsilon \xi \delta u\left(t+s_{1}\right), \ldots, \widetilde{u}\left(t+s_{k}\right)+\varepsilon \xi \delta u\left(t+s_{k}\right)\right)-\widetilde{f}_{u_{\nu+j}}(t) \mid\right] d \xi\right] d S\right\} d t,
\end{gathered}
$$




$$
\begin{gathered}
\sigma_{2}(\varepsilon \delta \mu)=\|Y\| \int_{0}^{\widetilde{T}+\delta_{0}}\left\{\int _ { S } \left[\int_{0}^{1} \sum_{j=1}^{s} \mid f_{x_{j}}\left(t, \widetilde{x}\left(\tau_{1}(t)\right)+\right.\right.\right. \\
+\xi \Delta x\left(\tau_{1}(t)\right), \ldots, \widetilde{x}\left(\tau_{s}(t)\right)+\xi \Delta x\left(\tau_{s}(t)\right), \widetilde{u}\left(\theta_{1}(t)\right)+\varepsilon \xi \delta u\left(\theta_{1}(t)\right), \ldots \\
\ldots, \widetilde{u}\left(\theta_{\nu}(t)\right)+\varepsilon \xi \delta u\left(\theta_{\nu}(t)\right), \widetilde{u}\left(t+s_{1}\right)+\varepsilon \xi \delta u\left(t+s_{1}\right), \ldots \\
\left.\left.\left.\ldots, \widetilde{u}\left(t+s_{k}\right)+\varepsilon \xi \delta u\left(t+s_{k}\right)\right)-\widetilde{f}_{x_{j}}(t) \mid d \xi\right] d S\right\} d t .
\end{gathered}
$$

It is not difficult to see that the convergence $\lim _{\varepsilon \rightarrow 0} \sigma_{i}(\varepsilon \delta \mu)=0, i=1,2$, is uniform for $\delta \mu \in V_{1}$.

Now,

$$
\begin{gathered}
\|\Delta x\| \leq \varepsilon\|\delta x(\delta \mu)\|+\|R(\varepsilon \delta \mu)\| \leq \\
\leq \varepsilon\left(\|\delta x(\delta \mu)\|+\sigma_{1}(\varepsilon \delta \mu)\right)+\sigma_{2}(\varepsilon \delta \mu)\|\Delta x\| .
\end{gathered}
$$

Here

$$
\|\delta x(\delta \mu)\|=\max _{0 \leq t \leq \widetilde{T}+\delta_{0}}|\delta x(t ; \delta \mu)|, \quad\|R(\varepsilon \delta \mu)\|=\max _{0 \leq t \leq \widetilde{T}+\delta_{0}}|R(t ; \varepsilon \delta \mu)| .
$$

Hence

$$
\|\Delta x\| \leq \frac{\varepsilon\left(\|\delta x(\delta \mu)\|+\sigma_{1}(\varepsilon \delta \mu)\right)}{1-\sigma_{2}(\varepsilon \delta \mu)}=\varepsilon \alpha(\varepsilon \delta \mu)
$$

where the value $\alpha(\varepsilon \delta \mu)$ is bounded when $\varepsilon \rightarrow 0$ uniformly for $\delta \mu \in V_{1}$.

Thus

$$
\|R(\varepsilon \delta \mu)\| / \varepsilon \leq \sigma_{1}(\varepsilon \delta \mu)+\alpha(\varepsilon \delta \mu) \sigma_{2}(\varepsilon \delta \mu)
$$

whence follows (21).

Let $D_{0}$ be a set of points $\zeta=\left(T, x_{0}, \varphi, u\right) \in I \times O_{1} \times G \times \Omega_{1}$ defined by the following condition: the system

$$
\begin{gathered}
\dot{x}(t)=\int_{S} f\left(t, x\left(\tau_{1}(t)\right), \ldots, x\left(\tau_{s}(t)\right), u\left(\theta_{1}(t)\right), \ldots\right. \\
\left.\ldots, u\left(\theta_{\nu}(t)\right), u\left(t+s_{1}\right), \ldots, u\left(t+s_{k}\right)\right) d S \\
x(t)=\varphi(t), \quad t \in[\tau, 0), \quad x(0)=x_{0}
\end{gathered}
$$

has the solution $x(t)=x(t ; \zeta)$ which is defined on $[0, T]$.

Thus the mapping

$$
L: D_{0} \rightarrow R^{1+n}
$$

is given on $D_{0}$ by the formula $L(\zeta)=\left(T, x_{0}, x(T)\right)$. It follows from Lemma 1 that the set $D_{0}$ is open in the topology induced from the space $E_{\zeta}=$ $R^{1} \times R^{n} \times E \varphi \times L_{\infty}$ 
Lemma 3. The mapping (24) is differentiable at the point $\widetilde{\zeta}=$ $\left(\widetilde{T}, \widetilde{x}_{0}, \widetilde{\varphi}, \widetilde{u}\right)$. Namely,

$$
\begin{gathered}
d L_{\widetilde{\zeta}}(\delta \zeta)=\left(\delta T, \delta x_{0}, \delta x_{1}\right), \\
\text { where } \delta x_{1}=\widetilde{f}(\widetilde{T}) \delta T+\delta x(\widetilde{T} ; \delta \mu), \delta \zeta=(\delta T, \delta \mu) \in E_{\zeta}-\widetilde{\zeta} .
\end{gathered}
$$

Proof. Let $V=\left\{\delta \zeta=(\delta T, \delta \mu)|| \delta T \mid \leq c, \delta \mu \in V_{1}\right\}$. With small enough $\varepsilon>0$ we have $\varepsilon \delta T \leq \delta_{0}$ for each $\delta \zeta \in V$. Therefore, using the representation (19) and the fact that $t=\widetilde{T}$ is the Lebesgue point of the function $\widetilde{f}(t)$, we have

$$
\begin{gathered}
x(\widetilde{T}+\varepsilon \delta T ; \varepsilon \delta \mu)=\widetilde{x}(\widetilde{T}+\varepsilon \delta T)+\varepsilon \delta x(\widetilde{T}+\varepsilon \delta T ; \delta \mu)+ \\
+R(\widetilde{T}+\varepsilon \delta T ; \varepsilon \delta \mu)=\widetilde{x}(\widetilde{T})+\int_{\widetilde{T}}^{\widetilde{T}+\varepsilon \delta T} \widetilde{f}(t) d t+\varepsilon \delta x(\widetilde{T} ; \delta \mu)+ \\
+\varepsilon(\delta x(\widetilde{T}+\varepsilon \delta T ; \delta \mu)-\delta x(\widetilde{T} ; \delta \mu))+R(\widetilde{T}+\varepsilon \delta T ; \delta \mu)= \\
=\widetilde{x}(\widetilde{T})+\varepsilon \delta x_{1}+o_{1}(\varepsilon \delta \zeta),
\end{gathered}
$$

where $\lim _{\varepsilon \rightarrow 0} o_{1}(\varepsilon \delta \zeta) / \varepsilon=0$ uniformly for $\delta \zeta \in V$.

Therefore

$$
\begin{gathered}
L(\widetilde{\zeta}+\varepsilon \delta \zeta)-L(\widetilde{\zeta})=\left(\widetilde{T}+\varepsilon \delta T, \widetilde{x}_{0}+\varepsilon \delta x_{0}, x(\widetilde{T}+\varepsilon \delta T)\right)- \\
-\left(\widetilde{T}, \widetilde{x}_{0}, \widetilde{x}(\widetilde{T})\right)=\left(\delta T, \delta x_{0}, \delta x_{1}\right) \cdot \varepsilon+o(\varepsilon \delta \zeta)=d L_{\widetilde{\zeta}}(\delta \zeta) \varepsilon+o(\varepsilon \delta \zeta),
\end{gathered}
$$

where $o(\varepsilon \delta \zeta)=\left(0,0, o_{1}(\varepsilon \delta \zeta)\right)$.

2.2. Deduction of the maximum principle. Consider the linear topological space $E_{z}=R^{1} \times E_{\zeta}$ of the points $z=(\sigma, \zeta)$.

Let $D$ be a subset of this space,

$$
D=\left\{z|z=(\sigma, \zeta)| \sigma \in R^{1}, \zeta \in D_{0}\right\} .
$$

The set $D$ is open (the more so, finitely open) (see $[2,3]$ ). Define on $D$ the mapping $P: D \rightarrow R^{1+l}$ by the formula

$$
P(z)=Q(L(\zeta))+(\sigma, 0, \ldots, 0)=\left(q^{o}(L(\zeta)), \ldots, q^{l}(L(\zeta))\right)+(\sigma, 0, \ldots, 0) .
$$

The differential of the mapping $P$ at the point $\widetilde{\zeta}=\left(0, \widetilde{T}, \widetilde{x}_{0}, \widetilde{\varphi}, \widetilde{u}\right)$ has the form

$$
\begin{aligned}
d P_{\widetilde{z}}(\delta z) & =\widetilde{\sigma}+\frac{\partial \widetilde{Q}}{\partial t} \delta T+\frac{\partial \widetilde{Q}}{\partial x_{0}} \delta x_{0}+\frac{\partial \widetilde{Q}}{\partial x_{1}} \delta x_{1}, \\
\widetilde{\sigma} & =(\delta \sigma, 0, \ldots, 0), \quad \delta z \in E_{z}-\widetilde{z} .
\end{aligned}
$$


Using the expressions (20) and (25), we obtain

$$
\begin{aligned}
d P_{\widetilde{z}}(\delta z)=\widetilde{\sigma} & +\left(\frac{\partial \widetilde{Q}}{\partial t}+\frac{\partial \widetilde{Q}}{\partial x_{1}} \widetilde{f}(\widetilde{T})\right) \delta T+\left(\frac{\partial \widetilde{Q}}{\partial x_{0}}+\frac{\partial \widetilde{Q}}{\partial x_{1}} Y(0 ; \widetilde{T})\right) \delta x_{0}+ \\
+ & \sum_{j=1}^{s} \frac{\partial \widetilde{Q}}{\partial x_{1}} \int_{\tau_{j}(0)}^{0} Y\left(\gamma_{j}(t) ; \widetilde{T}\right) \widetilde{f}_{x_{j}}\left(\gamma_{j}(t)\right) \dot{\gamma}_{j}(t) \delta \varphi(t) d t+ \\
& +\frac{\partial \widetilde{Q}}{\partial x_{1}} \int_{0}^{\widetilde{T}} Y(t ; \widetilde{T})\left[\sum_{j=1}^{\nu} \widetilde{f}_{u_{j}}(t) \delta u\left(Q_{j}(t)\right)+\right. \\
& \left.+\sum_{j=1}^{k} \int_{S} \widetilde{f}_{u_{\nu+j}}\left(t ; s_{1}, \ldots, s_{k}\right) \delta u\left(t+s_{j}\right) d S\right] d t .
\end{aligned}
$$

In the space $E_{z}$ let us define a filter by the following elements:

$$
W_{\widetilde{z}}=\left(R_{+}^{1} \cap V_{0}\right) \times V_{\widetilde{T}} \times V_{\widetilde{x}_{0}} \times\left(G \cap V_{\widetilde{\varphi}}\right) \times\left(\Omega_{1} \cap V_{\widetilde{u}}\right),
$$

where $V_{0}, V_{\widetilde{T}}, V_{\widetilde{x}_{0}}, V_{\widetilde{\varphi}}, V_{\widetilde{u}}$ are arbitrary convex neighborhoods of the points $0 \in R^{1}, \widetilde{T} \in\left(0, T_{0}\right), \widetilde{x}_{0} \in O_{1}, \widetilde{\varphi} \in G, \widetilde{u} \in \Omega_{1}$, correspondingly, $R_{+}^{1}=[0, \infty)$.

The filter $\Phi$ is convex (the more so, quasi-convex, (see [2,3]). It is evident that $[\Phi]=\Phi$, where $[\Phi]$ is the filter whose elements are the convex hulls of the elements of the filter $\Phi$. It follows from Lemma 1 that the mapping $P$ is continuous on $\Phi$ (see $[2,3]$ ).

The optimality of the element $\widetilde{\zeta}$ is equivalent to its extremality and the latter implies criticality of the mapping $P$ on $\Phi$, which is proved by the well-known method (see [2,3]).

Thus all the premises for the necessary criticality condition are fulfilled $[2,3]$.

Therefore there exist a nonzero vector $\pi=\left(\pi_{0}, \ldots, \pi_{l}\right)$ and an element

$$
\widetilde{W}_{\widetilde{z}}=\left(R_{+}^{1} \cap \widetilde{V}_{0}\right) \times \widetilde{V}_{\widetilde{T}} \times \widetilde{V}_{\widetilde{x}_{0}} \times\left(G \cap \widetilde{V}_{\widetilde{\varphi}}\right) \times\left(\Omega_{1} \cap \widetilde{V}_{\widetilde{u}}\right)
$$

of the filter $\Phi$ such that

$$
\pi d P_{\widetilde{z}}(\delta z) \leq 0, \quad \forall \delta z \in K\left(\widetilde{W}_{\widetilde{z}}-\widetilde{z}\right),
$$

where $K(W)$ is a cone stretched on the set $W$. It is easily seen that the condition $\delta z \in K\left(\widetilde{W}_{\widetilde{z}}-\widetilde{z}\right)$ is equivalent to the condition $\delta \sigma \in R_{+}^{1}, \delta T \in$ $R^{1}-\widetilde{T}, \delta x_{0} \in R^{n}-\widetilde{x_{0}}, \delta \varphi \in K\left(\widetilde{V}_{\widetilde{\varphi}}-\widetilde{\varphi}\right) \supset G-\widetilde{\varphi}, \delta u \in K\left(\widetilde{V}_{\widetilde{u}}-\widetilde{u}\right) \supset \Omega_{1}-\widetilde{u}$.

Using the expression for the differential $d P_{\widetilde{z}}(\delta z)$, assuming that $\delta u=0$, $\delta \varphi=0$, and taking into consideration that $\delta \sigma \in R_{+}^{1}, \delta T, \delta x_{0}$ may take 
arbitrary values, we obtain for $\pi_{0} \leq 0$

$$
\left\{\begin{array}{l}
\pi\left(\frac{\partial \widetilde{Q}}{\partial t}+\frac{\partial \widetilde{Q}}{\partial x_{1}} \widetilde{f}(\widetilde{T})\right)=0, \\
\pi\left(\frac{\partial \widetilde{Q}}{\partial x_{0}}+\frac{\partial \widetilde{Q}}{\partial x_{1}} Y(0 ; \widetilde{T})\right)=0 .
\end{array}\right.
$$

Assuming that in (26) $\delta t_{1}=0, \delta x_{0}=0, \delta u=0, \delta \sigma=0$ we obtain

$$
\sum_{j=1}^{s} \pi \frac{\partial \widetilde{Q}}{\partial x_{1}} \int_{\tau_{j}(0)}^{0} Y\left(\gamma_{j}(t) ; \widetilde{T}\right) \widetilde{f}_{x_{j}}\left(\gamma_{j}(t)\right) \dot{\gamma}_{j}(t) \delta \varphi(t) d t \leq 0, \quad \delta \varphi \in G-\widetilde{\varphi}
$$

If we assume $\delta t_{1}=0, \delta x_{0}=\delta \varphi=0, \delta \sigma=0$ we shall get

$$
\begin{gathered}
\pi \frac{\partial \widetilde{Q}}{\partial x_{1}} \int_{0}^{\widetilde{T}} Y(t ; \widetilde{T})\left[\sum_{j=1}^{\nu} \widetilde{f}_{u_{j}}(t) \delta u\left(\theta_{j}(t)\right)+\right. \\
\left.+\int_{S} \sum_{j=1}^{k} \widetilde{f}_{u_{\nu+j}}\left(t ; s_{1}, \ldots, s_{k}\right) \delta u\left(t+s_{j}\right) d S\right] d t \leq 0 .
\end{gathered}
$$

Let us introduce the notation

$$
\psi(t)=\pi \frac{\partial \widetilde{Q}}{\partial x_{1}} Y(t ; \widetilde{T}), \quad t \in[0, \widetilde{T}] .
$$

It is clear that $\psi(t)$ satisfies the system (6) and the condition

$$
\psi(\widetilde{T})=\pi \frac{\partial \widetilde{Q}}{\partial x_{1}} .
$$

We can now rewrite the condition (27) in the form

$$
\left\{\begin{array}{l}
\pi \frac{\partial \widetilde{Q}}{\partial t}=-\psi(\widetilde{T}) \widetilde{f}(\widetilde{T}), \\
\pi \frac{\partial \widetilde{Q}}{\partial x_{0}}=-\psi(0) .
\end{array}\right.
$$

Equations (31) and (32) give the conditions of transversality (9). Taking into account (30), we can rewrite inequalities (28), (29) in the form (7), (8). Thus Theorem 1 is completely proved.

3. Proof of Theorem 2. Let for $\widetilde{\zeta}=(\widetilde{\varphi}, \widetilde{u}) \in \Delta_{2}$ the conditions (11), (12) be fulfilled. Introducing the notation $\Delta x(t)=x(t ; \zeta)-x(x ; \widetilde{\zeta}), \zeta \in \Delta_{2}$ and taking into account (10), (13), and (14), we obtain

$$
\begin{gathered}
0=\psi\left(T_{0}\right) \Delta x\left(t_{0}\right)-\psi(0) \Delta x(0)=\int_{0}^{T_{0}} \frac{d}{d t}(\psi(t) \Delta x(t)) d t= \\
=\int_{0}^{T_{0}}\left[\dot{\psi}(t) \Delta x(t)+\psi(t) \frac{d}{d t} \Delta x(t)\right] d t=
\end{gathered}
$$




$$
\begin{gathered}
=\int_{0}^{T_{0}}\left\{\sum_{j=1}^{s} \psi_{0}\left(\gamma_{j}(t)\right) \widetilde{g}_{x_{j}}\left(\gamma_{j}(t)\right) \dot{\gamma}_{j}(t) \Delta x(t)-\sum_{j=1}^{s} \psi\left(\gamma_{j}(t)\right) A_{j}\left(\gamma_{j}(t)\right) \dot{\gamma}_{j}(t) \Delta x(t)+\right. \\
\left.+\psi(t) \sum_{j=1}^{s} A_{j}(t) \Delta x\left(\tau_{j}(t)\right)+\psi(t)[f(t, u(\cdot))-f(t, \widetilde{u}(\cdot))]\right\} d t
\end{gathered}
$$

Further, performing elementary transformations, in view of (14) we get

$$
\begin{aligned}
& 0=\psi\left(T_{0}\right) \Delta x\left(t_{0}\right)-\psi(0) \Delta x(0)=\sum_{j=1}^{s} \int_{0}^{\tau_{j}\left(T_{0}\right)} \tilde{g}_{x_{j}}\left(\gamma_{j}(t)\right) \dot{\gamma}_{j}(t) \Delta x(t) d t- \\
& -\sum_{j=1}^{s} \int_{0}^{\tau_{j}\left(T_{0}\right)} \psi\left(\gamma_{j}(t)\right) A_{j}\left(\gamma_{j}(t)\right) \dot{\gamma}_{j}(t) \Delta x(t) d t+ \\
& +\sum_{j=1}^{s} \int_{\tau_{j}(0)}^{\tau_{j}\left(T_{0}\right)} \psi\left(\gamma_{j}(t)\right) \times A_{j}\left(\gamma_{j}(t)\right) \dot{\gamma}_{j}(t) \Delta x(t) d t+ \\
& +\int_{0}^{T_{0}} \psi(t)[f(t, u(\cdot))-f(t, \widetilde{u}(\cdot))] d t=\sum_{j=1}^{s} \int_{\gamma_{j}(0)}^{T_{0}} \widetilde{g}_{x_{j}}(t) \Delta x\left(\tau_{j}(t)\right) d t+ \\
& +\sum_{j=1}^{s} \int_{\tau_{j}(0)}^{0} \psi\left(\gamma_{j}(t)\right) A_{j}\left(\gamma_{j}(t)\right) \dot{\gamma}_{j}(t) \Delta x(t) d t+\sum_{j=1}^{s} \int_{0}^{\gamma_{j}(0)} \widetilde{g}_{x_{j}}(t) \Delta x\left(\tau_{j}(t)\right) d t- \\
& -\sum_{j=1}^{s} \int_{\tau_{j}(0)}^{0} \widetilde{g}_{x_{j}}\left(\gamma_{j}(t)\right) \dot{\gamma}_{j}(t) \Delta x(t) d t+\int_{0}^{T_{0}} \psi(t)[f(t, u(\cdot))-f(t, \widetilde{u}(\cdot))] d t= \\
& =\sum_{j=1}^{s} \int_{0}^{T_{0}} \widetilde{g}_{x_{j}}(t) \Delta x\left(\tau_{j}(t)\right) d t+\sum_{j=1}^{s} \int_{\tau_{j}(0)}^{0}\left[\psi\left(\gamma_{j}(t)\right) A_{j}\left(\gamma_{j}(t)\right)-\right. \\
& \left.-\widetilde{g}_{x_{j}}\left(\gamma_{j}(t)\right)\right] \dot{\gamma}_{j}(t) \Delta x(t) d t+\int_{0}^{T_{0}} \psi(t)[f(t, u(\cdot))-f(t, \widetilde{u}(\cdot))] d t .
\end{aligned}
$$

Further,

$$
\begin{gathered}
I(\widetilde{\zeta})-I(\zeta)=\int_{0}^{T_{0}}\left[g\left(t, \widetilde{x}\left(\tau_{1}(t)\right), \ldots, \widetilde{x}\left(\tau_{s}(t)\right)\right)-g\left(t, x\left(\tau_{1}(t)\right), \ldots\right.\right. \\
\left.\left.\ldots, x\left(\tau_{s}(t)\right)\right)+f^{0}(t, \widetilde{u}(\cdot))-f^{0}(t, u(\cdot))\right] d t+\psi\left(T_{0}\right) \Delta x\left(T_{0}\right)- \\
-\psi(0) \Delta x(0)=\int_{0}^{T_{0}}\left[g\left(t, \widetilde{x}\left(\tau_{1}(t)\right), \ldots, \widetilde{x}\left(\tau_{s}(t)\right)\right)-g\left(t, x\left(\tau_{1}(t)\right), \ldots\right.\right. \\
\left.\left.\ldots, x\left(\tau_{s}(t)\right)\right)+\sum_{j=1}^{s} \widetilde{g}_{x_{j}}(t) \Delta x\left(\tau_{j}(t)\right)\right] d t+\sum_{j=1}^{s} \int_{\tau_{j}(0)}^{0}\left[-\widetilde{g}_{x_{j}}\left(\gamma_{j}(t)\right)+\right.
\end{gathered}
$$




$$
\begin{gathered}
\left.+\psi\left(\gamma_{j}(t)\right) A_{j}\left(\gamma_{j}(t)\right)\right] \dot{\gamma}_{j}(t)(\varphi(t)-\widetilde{\varphi}(t)) d t+\int_{0}^{T_{0}}\left\{-f^{0}(t, u(\cdot))+\right. \\
\left.+\psi(t) f(t, u(\cdot))-\left[-f^{0}(t, \widetilde{u}(\cdot))+\psi(t) f(t, \widetilde{u}(\cdot))\right]\right\} d t .
\end{gathered}
$$

Due to the convexity of the function $g$, by virtue of the inequalities (11), (12), it follows from $(33)$ that $I(\widetilde{\zeta})-I(\zeta) \leq 0$ for $\zeta \in \Delta_{2}$.

\section{REFERENCES}

1. G. L. Kharatishvili, Z. A. Machaidze, N. I. Markozashvili, and T. A. Tadumadze, Abstract variational theory and its applications to optimal problems with delays. (Russian) Metsniereba, Tbilisi, 1973.

2. R. V. Gamkrelidze and G. L. Kharatishvili, Extremal problems in linear topological spaces I. Math. Systems Theory 1 (1969), No. 3, 229256.

3. R. V. Gamkrelidze and G. L. Kharatishvili, Extremal problems in linear topological spaces. (Russian) Izv. Akad. Nauk SSSR, Ser. Math. 33 (1969), 781-839.

(Received 13.07.1994)

Authors' addresses:

G. Kharatishvili

Institute of Cybernetics

Georgian Academy of Sciences

5, S. Euli St., Tbilisi 380086

Republic of Georgia

T. Tadumadze

I. Vekua Institute of Applied Mathematics

Tbilisi State University

2, University St., Tbilisi 380043

Republic of Georgia 